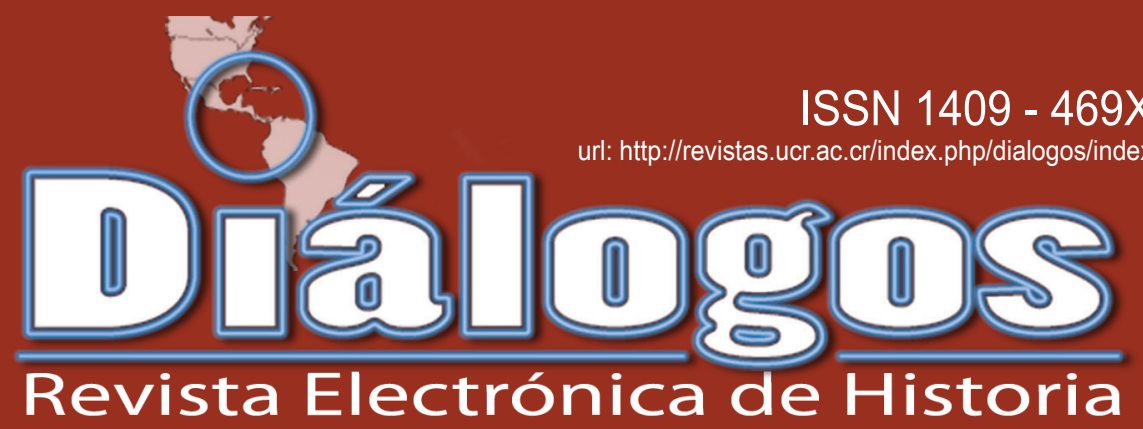

Escuela de Historia. Universidad de Costa Rica Volumen 14 especial en homenaje a Victor Hugo Acuña. Octubre 2013

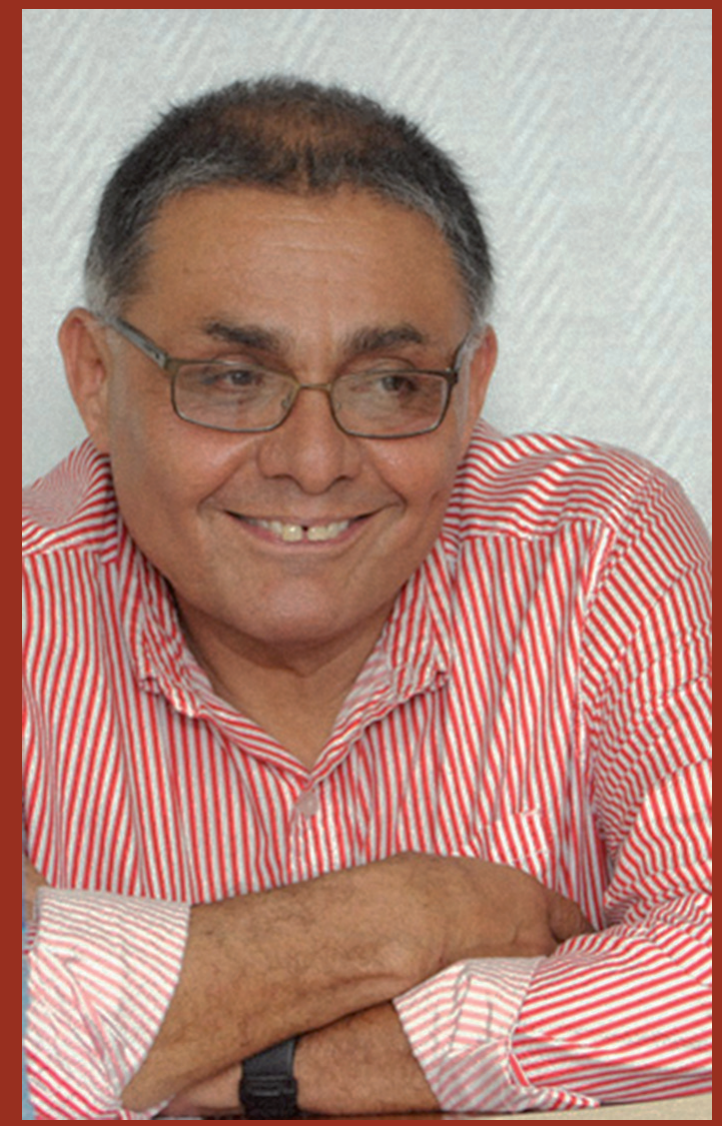

Director de la Revista: Dr. Juan José Marín Hernández juan.marinhernandez@ucr.ac.cr

Editor académico: Dr. Ronny Viales Hurtado - ronny.viales@ucr.ac.cr Editor técnico: M.Sc. Marcela Quirós G. - marcela.quiros@ucr.ac.cr 



\section{Miembros del Consejo Editorial:}

Dr. Juan José Marín Hernández, Catedrático. Director del Centro de Investigaciones Históricas de América Central. Universidad de Costa Rica. Costa Rica. juan. marin@ucr.ac.cr

Dr. Ronny Viales Hurtado. Catedrático. Historia Económica y Social. Universidad de Costa Rica. Director de la Escuela de Historia. Costa Rica. ronny. viales@ucr.ac.cr

Dr. David Díaz Arias: Catedrático. Historia Política, Director del posgrado de Historia y Docente de la Escuela de Historia, Universidad de Costa Rica, Costa Rica.david.diaz@ucr.ac.cr

MSc. Francisco Enríquez. Historia Social. Universidad de Costa Rica. Costa Rica. francisco.enriquez@ucr. ac.cr

Dra. Ana María Botey. Historia de los movimientos sociales. Universidad de Costa Rica. Costa Rica. abotey@gmail.com

\section{Miembros del Consejo Asesor Internacional:}

Dr. José Cal Montoya. Universidad de San Carlos de Guatemala.Guatemala. jecalm@correo.url.edu.gt

Dr. Juan Manuel Palacio. Universidad Nacional de San Martín. Argentina.jpalacio@unsam.edu.ar

Dr. Eduardo Rey. Universidad de Santiago de Compostela. España. ereyt@usc.es

Dr. Heriberto Cairo Carou. Departamento de Ciencia Política y de la Administración III Universidad Complutense de Madrid. España. hcairoca@cps.ucm.es

Dra. Rosa de la Fuente. Departamento de Ciencia Política y de la Administración III Universidad Complutense de Madrid. España. rdelafuente@cps. ucm.es

Dr. Javier Franzé. Departamento de Ciencia Política y de la Administración III Universidad Complutense de Madrid. España. javier.franze@cps.ucm.es

Dr. Jaime Preciado Coronado Departamento de Estudios Ibéricos y Latinoamericanos. Universidad de Guadalajara. México. japreco@hotmail.com
Dr. Gerónimo de Sierra. Vicerrector de la Universidade Federal da Integração Latino-Americana (UNILA) y Departamento de Sociología, Facultad de Ciencias Sociales de la Universidad de la República. Uruguay. geronimo@fcs.edu.uy

Dr. Antonio Palazuelos. Departamento de Ciencia Política y de la Administración III - Universidad Complutense de Madrid. España. palazuelosa@cps. ucm.es

Dr. Werner Mackenbach. Universidad Potsdam. Alemania. werner.mackenbach@uni-potsdam.de

Dr. Guillermo Castro. Ciudad del Saber Panamá. Panamá.gcastro@cdspanama.org

Dra. Natalia Milanesio. University of Houston. Estados Unidos.nmilane2@Central.UH.EDU

Dr. Ricardo González Leandri. Consejo Superior de Investigaciones Científicas - España. España. rgleandri@gmail.com

Dra. Mayra Espina. Centro de Estudios Psicológicos y Sociológicos, La Habana. Cuba.mjdcips@ceniai.inf.cu

Dra. Montserrat Llonch. Departamento de Economía e Historia Económica Universidad Autónoma de Barcelona. España.montserrat.llonch@uab.es

Dra. Estela Grassi. Universidad de Buenos Aires. Argentina. estelagrassi@gmail.com

Dra. Yolanda Blasco. Universidad de Barcelona. España. yolandablasco@ub.edu

Dr. Alfredo Falero. Departamento de Sociología. Universidad de la República. Uruguay. alfredof@adinet. com.uy

\section{Portada:}

Fotografía de Anel Kenjekeeva, Oficina de Divulgación Universidad de Costa Rica, publicada en http://www. ucr.ac.cr/noticias/2012/09/13/escuela-de-historia-rindehomenaje-a-victor-hugo-acuna.html

\section{Equipo Técnico Editorial:}

$\begin{array}{ll}\text { Editora Técnica: } & \text { M.Sc. Marcela Quirós Garita. } \\ & \text { marcela.quiros@ucr.ac.cr } \\ \text { Diagramación: } & \text { Cindy Chaves Uribe } \\ \text { Asistentes: } & \begin{array}{l}\text { Pablo Hurtado Granados } \\ \text { Maureen Méndez Montero }\end{array}\end{array}$


"Diálogos Revista Electrónica de Historia" se publica desde octubre de 1999.

\section{Diálogos está en los siguientes repositorios:}

Dialnet

http://dialnet.unirioja.es/servlet/

revista?tipo_busqueda=CODIGO\&clave_revista $=3325$

Latindex

http://www.latindex.unam.mx/larga.php?opcion=1\&folio=12995;

\section{UCRindex}

http://www.revistas.ucr.ac.cr/

\section{Scielo}

http://www.scielo.cl/

\section{eRevistas}

http://www.erevistas.csic.es/

\section{REDALYC}

http://redalyc.uaemex.mx/src/inicio/FrmBusRevs2.jsp?iEdoRev=2\&cvepai=11;

\section{LANIC}

http://lanic.utexas.edu/la/ca/cr/indexesp.html

Repositorio de Revistas Universidad de Costa Rica http://www.latindex.ucr.ac.cr/

Directorio y recolector de recursos digitales del

Ministerio de Cultura de España

http://roai.mcu.es/es/inicio/inicio.cmd

DOAJ Directory of open access \& Hybrid journals

http://www.doaj.org/doaj?func=byTitle\&hybrid=1\&query=D

Biblioteca de Georgetown

http://library.georgetown.edu/newjour/d/msg02735.html

Asociación para el Fomento de los Estudios Históricos

en Centroamérica

http://afehc. apinc.org/index.php?action=fi_aff\&id=1774

Universidad de Saskatchewan, Canadá

https://library.usask.ca/ejournals/view/1000000000397982

\section{Monografias}

http://www.monografias.com/Links/Historia/more12.shtml

\section{Hispanianova}

http://hispanianova.rediris.es/general/enlaces/hn0708.htm

Universidad del Norte, Colombia

http://www.uninorte.edu.co/publicaciones/memorias/enlaces.htm

Universidad Autónoma de Barcelona

http://seneca.uab.es/historia/hn0708.htm

Repositorio Invenia - Gestión del Conocimiento

http://www.invenia.es/oai:dialnet.unirioja.es:ART0000086144

\section{Enlace Académico}

http://www.enlaceacademico.org/biblioteca/

revistas-en-formato-digital-centroamerica/

\section{Electronic Resources}

http://sunzi1.lib.hku.hk/ER/detail/hkul/3987318

Revistas académicas en texto completo

http://web.prw.net/ vtorres/
Diálogos se anuncia en las siguientes

instituciones y sitios académicos:

Maestroteca

http://www.maestroteca.com/detail/553/dialogos-revista-electronica-de-historia.html

Biblioteca de Georgetown

http://library.georgetown.edu/newjour/d/msg02735.html

Asociación para el Fomento de los Estudios Históricos en Centroamérica

http://afehc. apinc.org/index.php?action=fi_aff\&id=1774

Universidad de Saskatchewan, Canadá

https://library.usask.ca/ejournals/view/1000000000397982

\section{Monografias}

http://www.monografias.com/Links/Historia/more12.shtml

\section{Hispanianova}

http://hispanianova.rediris.es/general/enlaces/hn0708.htm

Universidad del Norte, Colombia

http://www.uninorte.edu.co/publicaciones/memorias/enlaces.htm |

Universidad Autónoma de Barcelona

http://seneca.uab.es/historia/hn0708.htm

Repositorio Invenia - Gestión del Conocimiento

http://www.invenia.es/oai:dialnet.unirioja.es:ART0000086144

\section{Enlace Académico}

http://www.enlaceacademico.org/biblioteca

revistas-en-formato-digital-centroamerica/

\section{Electronic Resources}

http://sunzi1.lib.hku.hk/ER/detail/hkul/3987318

Revistas académicas en texto completo

http://web.prw.net/ vtorres/

La revista electrónica Diálogos es financiada por

Vicerrectoría de Investigación de la Universidad de Costa Rica
Dialnet - eRevistas

- UCRindex - Latindex -

REDALYC - DOJAC - Directorio

y recolector de recursos

digitales del Ministerio de

Cultura de España

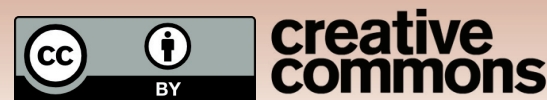





\title{
CONSIDERACIONES SOBRE EL TURISMO EN EL BARRIO PACUARE DE LIMÓN: DATOS Y REFLEXIONES PRELIMINARES
}

\section{CONSIDERATIONS ON TOURISM: BARRIO PACUARE DE LIMÓN PRELIMINARY DATA AND REFLECTIONS.}

Pablo Carballo Chaves

\author{
Palabras clave \\ Turismo, tipología laboral, Limón, Barrio Pacuare, Centros Históricos, economía, \\ problemas ambientales. \\ Keywords \\ Tourism, labor typology, Limón, Barrio Pacuare, historic centers of tourism , local economy, \\ environmental issues.
}

Fecha de recepción: 17 de mayo, 2013 - Fecha de aceptación: 8 de agosto, 2013

\begin{abstract}
Resumen
Esta ponencia/artículo expone tres consideraciones a partir de la construcción previa de un Censo en el Barrio Pacuare, ubicado en el Cantón central de Limón, Costa Rica. En el caso de las dos primeras consideraciones se exponen ideas relativas al turismo y las variables de edad y sexo de los participantes en las áreas establecidas según la tipología laboral propuesta, con el fin de comparar (más allá de este documento) la situación del trabajo en el turismo con otros sectores determinados como: público, formal, informal-doméstico, otros y no ocupados. La tercera consideración aborda el tema de las opiniones de personas vinculadas o no con la actividad, sobre el turismo, principalmente en los rubros de limitación de turistas y en lo concerniente a la influencia positiva o negativa para el barrio y en general, para la provincia de Limón. Estas tres consideraciones, a partir de los datos obtenidos, permiten entrever una reflexión inicial sobre la importancia práctica que tiene el turismo en términos de empleo y sobrevivencia, así como en la construcción simbólica del turismo/turista no-limitado (desde códigos estatales, por ejemplo) y la disposición a preferir las consecuencias generadas por la presencia masiva de turistas, a las derivadas del fenómeno contrario, o sea, la disminución en la afluencia turística. Lo anterior, manifiesta una prevalencia del tema económico sobre otros intereses sociales, ya que, pese a reconocer y valorar problemáticas de tipo ambiental, entre otras, el presupuesto económico condiciona las decisiones y los apoyos respecto a la actividad turística.
\end{abstract}

\begin{abstract}
This paper/article discusses three considerations from the previous census variables in the Pacuare neighborhood, located in Limon central county, Costa Rica. The first two considerations are ideas set about tourism and the variables of age and sex in certain areas established. According to the labor typology, in order to compare (beyond this document) tourism employment status with other employment areas (that complete the typology) such as: public, formal, informal-domestic
\end{abstract}


and not-occupied. Last consideration addresses the opinions of the persons associated or not with tourism activity. On tourism, mainly in the areas of limitation tourists and regarding the positive or negative influence for the neighborhood. These three data considerations, provide a glimpse of an initial reflection on the practical importance of tourism in terms of employment and survival. And in the symbolic construction of tourism/tourist not limited (from state codes, for example) and the disposition to prefer the consequences generated by the massive presence of tourists, to those derived from the opposite phenomenon, the decrease in tourist inflow. While people recognize and value environmental issues, among others, economic budget still leads and supports decisions regarding tourism. 


\section{CONSIDERACIONES SOBRE EL TURISMO EN EL BARRIO PACUARE DE LIMÓN: DATOS Y REFLEXIONES PRELIMINARES.}

Pablo Carballo Chaves

\section{INTRODUCCIÓN METODOLOGICA}

En el marco del proyecto de la Universidad de Costa Rica, denominado Centros Históricos del Turismo: Los casos de Puntarenas y Limón ${ }^{1}$, se llevaron a cabo diversas actividades relativas al estudio de la dinámica turística en espacios socio-geográficos de los pueblos costarricenses. Dentro de esta variedad de actividades se realizó un censo en un barrio central de Limón llamado Pacuare. Dicho censo se aplicó mediante una encuesta diseñada para valorar de entrada la diversidad y ubicación laboral de las personas, tanto en el nivel individual, como también involucrando la reflexión desde las unidades de hogares. Gracias a la ayuda de estudiantes de la Universidad de Costa Rica se realizó el censo que culminó en el 2011, el cual se procesó en la base de datos SPSS (Statistical Package for Social Sciences) en el 2012. Durante el procesamiento de datos se terminó por construir una tipología laboral que permitió, entre otras aproximaciones, una reflexión comparativa entre el turismo y otras actividades. Esta tipología contiene los siguientes segmentos: A. Turismo, B. Sector Público, C. Sector Formal, y D. Sector Informal Doméstico; a los cuales se les adjuntan otras dos categorías: E. Otros ${ }^{2}$ y F. No ocupados ${ }^{3}$, complementar los datos y las cantidades dentro del proceso de clasificación.

La anterior es una introducción sucinta del complejo abordaje metodológico que significó la elaboración del censo, así como de su preparación, ejecución y análisis. Lo que interesa tener como base de conocimiento sobre el procedimiento y el método utilizado, es que los datos presentados en este artículo surgen de la recolección de material a nivel censal y que, partiendo de dichos resultados, los análisis preliminares aquí expuestos son un grupo de interpretaciones que buscan orientar la lectura de ese material. En esta medida, son una guía que orienta, pero que no pretende condicionar la interpretación, sino abrir un debate a partir de una lectura sociológica.

También es importante indicar que el material empírico y sus reflexiones, elementos base del proyecto, fueron hechos a partir de la vinculación de diferentes entes de la Universidad de Costa Rica, es decir, el proyecto se concibió bajo un trabajo intersedes, en el que la Escuela de Sociología, la Sede de Limón y la Sede de Puntarenas interactuaron, para llevar a cabo el proyecto propuesto, así como los productos académicos particulares que surgieron de él. 


\title{
BREVE CONTEXTUALIZACIÓN DEL TURISMO EN COSTA RICA Y EL CARIBE
}

La inversión de capitales se ha masificado de manera creciente en espacios particulares de las dinámicas comerciales, tanto de productos, como de servicios. En este sentido, el turismo ha sido uno de los espacios donde la proyección en inversión ha crecido desde hace ya varias décadas, y con ello, la modificación de espacios y relaciones sociales ligadas con las áreas geográficas de las que se usufructúa la belleza paisajística y recursos específicos, entre otros elementos. La actividad tuvo un importante impulso durante los años noventa en Centroamérica, y específicamente en Costa Rica, por eso Pérez Sáinz, Andrade-Eekhoff y Bastos (2004) señalan:

\begin{abstract}
...hay que tomar en cuenta el turismo. Su dinamismo se refleja tanto en el hecho que en 1990 ingresaron en la región millón y medio de turistas, flujo que se incrementó en casi un millón más, siete años más tarde. Destacan dos países, Guatemala y Costa Rica. Pero si el primero de ellos, a inicios de la década, recibía un poco más de medio millón de visitantes, superando levemente al segundo, ya para 1997, Costa Rica había alcanzado los 800,000 turistas, cifra superior a la guatemalteca (similar a la de inicio de la década)

(CANATUR, 1998).
\end{abstract}

En Costa Rica se desarrollo esta actividad con más consistencia durante la década de los ochenta. Posteriormente, en los 90, tuvo su momento de auge masivo y global, ya que en esta época creció, pero esta vez con un amplio margen de inversión extranjera. En los años 50 y 60 se presentó una lógica de avance de la actividad asociada a lo que se denominó turismo interno ${ }^{4}$. De ahí que, una de las justificaciones del proyecto fue rescatar estos clásicos destinos turísticos nacionales, valorar sus circunstancias (que se explicitaran más adelante), y generar una serie de datos que colaboren con la comprensión del fenómeno del turismo en su condición económica y socio-cultural.

El encadenamiento del comercio y la productividad mundial incorporó al turismo dentro de esta dimensión de la capitalización de los espacios de ocio, en distintas facetas. Y también se aprovechó de distintos escenarios y servicios para construir áreas donde se establece un turismo que cumpla con estándares de satisfacción internacional6. La provincia de Limón se presenta como un ejemplo de esta penetración del capital internacional y la adecuación de sus variadas zonas, sociales y naturales, para albergar, además, del ya mencionado turismo local, la entrada de extranjeros en diferentes niveles de cantidades y actitudes. La provincia de Limón, dentro de su recorrido histórico-social, ha tenido diferentes incursiones en términos de participación de capitales internacionales, las cuales han causado muchas problemáticas sociales y el turismo se ha convertido en una más, pues no solo ha logrado convertirse en un mecanismo de ingresos, sino que, sin cuestionar 
las ventajas económicas que ofrece, ha deparado consecuencias y la consiguiente valoración de la actividad turística que hacen los pobladores de esta zona.

Varios pueden ser los puntos por analizar en el escenario del turismo en la zona, sin embargo podemos puntualizar en el aspecto jurídico/ambiental (García Saura \& Lepe Jorquera, 2009) y de aprovechamiento económico-comercial (Román \& Jiménez et.al, 2004) como dos de las facetas que han tenido mayor atención en los últimos años, y que forman parte del papel crítico que desempeña esta actividad en la provincia, así como en los niveles nacional e internacional. Dentro del desarrollo de la región se encuentra el ámbito de la desigualdad socio-cultural, históricamente presente, como indica Cordero Ulate (2011):

\begin{abstract}
La atracción de Limón como destino turístico no ha estado exenta de contradicciones prejuicios muy fuertes y arraigados entre la población blanca y mestiza del resto del país, especialmente la del Valle Central. Antes de 1949 a los habitantes negros de la provincia se les prohibía el paso hacia el centro del país, impidiéndoles viajar después de Turrialba, en dirección hacia San José. Simbólicamente debe destacarse que fue hasta 1964 que se agregó una estrella al escudo nacional, en representación justamente de Limón; la sétima provincia.
\end{abstract}

Así se mantiene un perfil crítico de las dificultades que presenta la región en un sentido más completo, que abarca otros aspectos además del turístico, aunque este sea el punto de atención del artículo.

\title{
EL ESPACIO DE LA COMUNIDAD Y LO GLOBAL
}

Una primera consideración surge al pensar teóricamente el espacio donde se llevó a cabo el estudio, ya que al ser un barrio -Pacuare de Limón-, esta condición sugiere una condensación de dinámicas sociales, en las cuales se anclan diferentes actividades "nuevas" o re-significadas, dentro de ese espacio o espacios/ comunidades ya existentes. Aquí es fundamental tener claro que no estamos solamente estudiando el turismo, sino que el turismo interactúa en dinámicas sociales ya previamente configuradas, donde no solamente se instala, sino que participa en adaptaciones que no necesariamente son eficientes, a lo interno de la misma comunidad.

En este sentido, podemos aportar que la tradición de la idea conceptual de comunidad es ya de larga data para la Sociología, y que esta reflexión clásica no está exenta de problemáticas analíticas y prácticas. Desde la diferencia que establecía Ferdinand Tönnies entre sociedad y comunidad ${ }^{6}$, el tema de la comunidad ha estado asociado a los elementos de lazos de familiaridad, cercanía, amistad y vínculos históricos. En otras líneas, también se puede rescatar el carácter relacional 
con el nacionalismo (Poviñan, 1949), sin embargo no entraremos en esta discusión. Una posibilidad reflexiva es entrar a las actividades laborales con énfasis en el turismo a partir de una lógica de zona/barrio, que se ancla en una configuración, que podríamos señalar, como de comunidad(es); siguiendo los elementos señalados por Tönnies ${ }^{7}$ y otros exponentes de la materia, sin entrar de lleno en un estudio temático.

Los nuevos patrones globales de mercado y sociedad hacen replantear el papel de las comunidades en dicho entramado mundial, máxime que el turismo, dentro de la dimensión capitalista de acumulación, se ha posicionado con fuerza en sitios que no tienen, necesariamente, la tradición de globalidad de otros. En este sentido, Alejandro Bialakowsky apunta: "En Giddens, la comunidad estará relacionada al problema del registro reflexivo y la reflexividad institucional en aumento tanto en la temprana modernidad, como aún más en condiciones de modernidad tardía. Las comunidades que emergen o re-emergen sólo podrán ser comprendidas si se establecen las correlaciones necesarias con los conceptos de saber mutuo y agencia" (2010). Por lo tanto, lo que ha ocurrido es la incursión socio-económica de una comunidad en una dinámica económico-comercial, que no necesariamente se ha "controlado" internamente, ya que el aspecto global ha incidido de maneras complejas y ambiguas en el entramado de esta forma de comunidad.

El turismo se presenta dentro de factores emergentes de la lógica de mercado capitalista, y es fundamental en el espacio socio-laboral, donde lo natural, lo cultural, y lo social, en general, entran como recursos de explotación o recursos de apoyo, para proporcionar experiencias de ocio en diferentes medidas y contextos. Así que, en muchas oportunidades, la organización de la vida en las comunidades se re-crea para satisfacer requerimientos de ocio externos, y que terminan por moldear y acondicionar la propia población, debido a las demandas de turistas y de inversión, que intervienen también en la reconstrucción socio-estructural de las regiones. $\mathrm{La}$ actividad turística participa, cada vez con más fuerza, en la estructuración de las comunidades limonenses, e incluso se podría hablar de la sociedad limonense en general, que además está permeada por la lógica desarrollista del Estado costarricense promovida durante los años setenta y ochenta, durante los cuales el apogeo formalista captó importantes grupos de personas, pero que en la actualidad se ha encontrado en un estancamiento riesgoso y preocupante. Lo anterior, ha obligado a los funcionarios, junto con aquellos que nunca ingresaron en las filas del Estado, a buscar trabajos en lo privado y, en muchos de los casos, en el sector informal, opción que se presenta con más fuerza en los últimos años.

Al estudiar un barrio de una zona en particular podemos vislumbrar cierta idea de patrones o circunstancias relativas a lazos específicos de convivencia humana, tal y como, en un nivel básico, es sobrevivir ${ }^{8}$, y que además permite explicar ciertas lógicas de comportamiento y de acomodos, en términos de la apropiación 
de una dinámica laboral, aplicado en este caso, al Turismo en Limón. En esta línea podemos prever desproporción de tarifas, salarios, contrataciones ambiguas, así como también problemas de impacto cultural en prácticas de personas locales y foráneas, así como el dilema de la adaptación o la posible imposición por parte de unos u otros sobre las formas de comportamiento. Es así como desde ya podemos entrever un reacomodo en dinámicas económico-comerciales, que han terminado por modificar conductas culturales, de tal manera que han creado fracturas socioculturales 9 .

La comunidad es entonces, en lo que concierne en este momento, una relación que preserva rasgos de convivencia histórica y vivencial, en una zona o espacio socio-geográfico particular y que entra en diálogo directo con las personas que lo habitan, moldeándose mutuamente. Además, con un nivel de agencia (relativo), con el cual se pueda reconstruir la vivencia y la apropiación de las relaciones, recursos y perspectivas sociales. Sin embargo, complementada con la modernidad y los impulsos capitalistas, la comunidad es un espacio de confrontación y debate, donde viejos y nuevos paradigmas socio-económicos se desarrollan, y grupos distintos, dentro y fuera de ella, establecen criterios para la apropiación y uso de los recursos que posee en general, y en específico los socio-geográficos que se pueden aprovechar, en la mayoría de los casos, desde la mirada económico-comercial.

\section{CONSIDERACIONES INICIALES Y ANÁLISIS A PARTIR DE LOS DATOS}

En primer lugar es importante aclarar elementos de carácter metodológico. En el censo hecho durante el 2011 se logró recopilar información individual sobre la población estudiada del Barrio Pacuare. En dicha sección, según los datos individuales de las personas, se obtuvo información como edad y escolaridad, entre otros. En la Tabla 1 se presentan las cantidades de habitantes entrevistados, según las categorías de la tipología; mientras que en la Tabla 2 se muestra información más detallada sobre las variables claves respecto a los individuos y las respectivas categorías, tanto en promedios como en porcentajes.

En la tabla de la tipología laboral de individuos (Tabla 1) se muestra la presencia de las áreas de ocupación en el sector público y formal, según la captura de información. Es así como el sector formal es el ámbito donde se logró detectar más personas, lo cual refleja, de primera entrada, la importancia del sector en la dinámica de trabajo del barrio. En la tabla de tipología de hogares (Tabla 2) se presenta la distribución de datos según características de las personas, especificando también el carácter de sus particularidades laborales.

En el caso de la consideración sobre la tipología de individuos, partimos de que las personas ubicadas en el sector turismo muestran una de las variables 
TABLA 1. TIPOLOGÍA LABORAL DE LA BASE DE INDIVIDUOS EN EL BARRIO PACUARE, SEGÚN SU NÚMERO DE ENTREVISTADOS EN ÁREA DE ACTIVIDAD. 2011-2012.

\begin{tabular}{cccccc}
\hline Turismo & Público & Formal & $\begin{array}{l}\text { Informal- } \\
\text { Doméstico }\end{array}$ & Otros & Total \\
N: 34 & N: 76 & N: 95 & N: 52 & N: 19 & N: 276 \\
\hline
\end{tabular}

Fuente: Elaboración propia en el marco del proyecto Centros Históricos del Turismo. Censo 2011.

TABLA 2. TIPOLOGÍA LABORAL DE LA BASE DE HOGARES EN EL BARRIO PACUARE, SEGÚN SU NÚMERO DE ENTREVISTADOS SEGÚN ÁREA DE ACTIVIDAD.

\begin{tabular}{ccccccc}
\hline Turismo & Público & Formal & $\begin{array}{c}\text { Informal-Do- } \\
\text { méstico }\end{array}$ & Otros & $\begin{array}{c}\text { No } \\
\text { Ocupado }\end{array}$ & Total \\
N: 29 & N: 54 & N: 64 & N: 38 & N: 42 & N: 54 & N: 281 \\
\hline
\end{tabular}

Fuente: Elaboración propia en el marco del proyecto Centros Históricos del Turismo. Censo 2011.

más bajas en la edad, y la tipología informal-doméstica es la más alta (el "otros" mezcla diferentes elementos, por tanto el promedio de 45 es sumatorio de varios rubros). Además, la edad de la escolaridad en el caso de la actividad turística es de 9.03 y se equipara fuertemente con el caso del sector Formal de 9.06. Una tercera variable a considerar es el sexo de los trabajadores del turismo, pues se demuestra que la mayoría son mujeres, en un $61.8 \%$. Estos tres elementos son recuperados en la Tabla 3 y se utilizan para desarrollar esta primera consideración. 
TABLA 3. VARIABLES INDIVIDUALES DEL BARRIO PACUARE, SEGÚN LA TIPOLOGÍA LABORAL CONSTRUIDA. (PROMEDIOS Y PORCENTAJES) 2011-2012.

\begin{tabular}{|c|c|c|c|c|c|c|}
\hline Variables seleccionadas & Turismo & Público & Formal & $\begin{array}{l}\text { Informal- } \\
\text { Doméstico }\end{array}$ & Otros & Total \\
\hline Edad (Promedio en años) & 37.62 & 39.46 & 37.27 & 43.21 & 45.32 & 39.59 \\
\hline $\begin{array}{l}\text { Escolaridad (Promedio en } \\
\text { años) }\end{array}$ & 9.03 & 11.87 & 9.06 & 8.29 & 7.78 & 9.60 \\
\hline $\begin{array}{l}\text { Antigüedad Laboral (Pro. en } \\
\text { años) }\end{array}$ & 7.06 & 11.42 & 5.60 & 9.06 & 13.32 & 8.57 \\
\hline $\begin{array}{l}\text { Horas de trabajo a la semana } \\
\text { (pro.) }\end{array}$ & 48.7 & 45.4 & 53.6 & 40.1 & 56.4 & 48.3 \\
\hline $\begin{array}{l}\mathrm{N}^{\circ} \text { personas en el estableci- } \\
\text { miento }\end{array}$ & 16.8 & 865.7 & 106.8 & 1.52 & 1.67 & 192.4 \\
\hline Sexo (\% Hombres) & 38.2 & 47.4 & 72.6 & 28.8 & 52.6 & 51.8 \\
\hline $\begin{array}{l}\text { Cotizan al seguro social (\% } \\
\text { SI) }\end{array}$ & 52.9 & 96.1 & 94.7 & 3.8 & 94.4 & 72.7 \\
\hline Recibe capacitación (\% NO) & 76.5 & 28.0 & 57.9 & 78.4 & 78.9 & 57.3 \\
\hline $\begin{array}{l}\text { Localización Estableci- } \\
\text { miento }\end{array}$ & & & & & & \\
\hline (\% mismo cantón) & 81.8 & 95.7 & 90.4 & 94.2 & 94.7 & 91.8 \\
\hline $\begin{array}{l}\text { El dueño es del propio } \\
\text { cantón }(\% \mathrm{SI})\end{array}$ & 59.3 & 100 & 37.6 & 87.2 & 94.1 & 59.6 \\
\hline
\end{tabular}

Fuente: Elaboración propia en el marco del proyecto Centros Históricos del Turismo. Censo 2011. 
La consideración radica en analizar que el turismo es, o se ha convertido, en un escenario laboral fundamental para personas que condensan (o por aparte) las dimensiones de edades de trabajo, y que podrían estar en situación de dependencias familiares, además cabría mencionar las jefaturas familiares (como lo contrastaremos más adelante), ya que la mayoría son mujeres. Por otra parte, no son personas que cuentan, necesariamente, con baja escolaridad, en comparación con otros sectores de la tipología, lo que permite realizar algunas acciones de vínculos con el sector turístico. En otras palabras, el turismo es un escenario posicionado como un trabajo que capta individuos de un perfil social específico: femenino y joven, que no encuentra espacio en la normativa socio-empleadora pública de la región, según los datos obtenidos.

Esta primera consideración, si bien surge de una valoración de datos de individuos, es importante que se analice dentro de una lógica de hogares, ya que esto permite abordar no solo al sujeto que trabaja en el turismo, sino también, a quienes dependen, indirectamente, de la actividad. Esta gama de datos se presenta en la Tabla 4.

A partir de esta tabla aportamos una segunda consideración, la cual consiste en señalar que la jefatura de los hogares se posiciona de manera significativa en las mujeres. Esta condición coincide con la anterior, que señala los condicionantes de sexo y edad: mujeres y juventud, en el caso de personas contratadas en el sector turismo. Por otra parte, podemos agregar que el turismo, además de convertirse o ser una fuente de ingreso fundamental, se posiciona en una condición salarial mucho mejor que otros sectores, tal es el caso del informal-doméstico, en el que se ubica otra proporción de la población laboral femenina.

En resumen, esta segunda consideración señala que el turismo, además de ser un escenario social de posibilidades de manutención, cuando se estudian los perfiles de las personas que acuden a este espacio laboral, queda demostrada la importancia que tiene para la dinámica familiar, ya que los hogares están encabezados, en buena medida, por mujeres. Esto no quiere decir que el turismo sea la única opción de trabajo en que se podrían desempeñar, pero si se posiciona como un factor relevante. Esta particularidad puede estar contribuyendo a una adaptación, en términos de roles de género.

En este sentido, las dos primeras consideraciones, si bien es cierto, se unifican en el criterio analítico de estudiarlas desde la participación de las personas jóvenes y las jefaturas de familia femeninas, es importante puntualizar en que la segunda reflexión se basa en ese razonamiento para fundamentar una posible modificación o adaptación, según se presente, en los roles de género, es decir, un cuestionamiento más estructural a las bases relacionales de las comunidades y los patrones socio-laborales.

La tercera y última consideración preliminar, a partir de los datos obtenidos, es relativa a las opiniones de las personas de la comunidad de Pacuare sobre el 


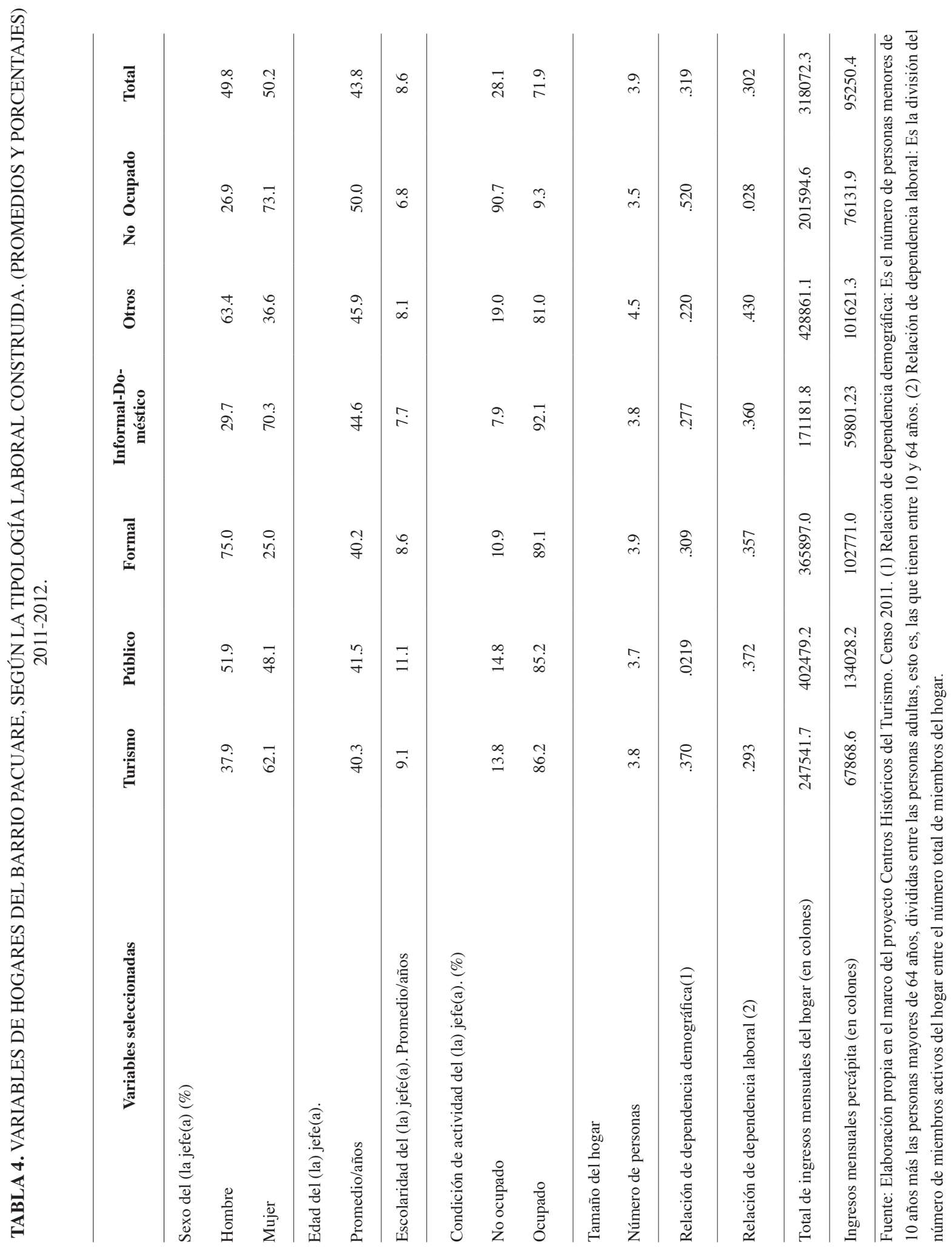


turismo (véase la Tabla 5). En este sentido, es importante rescatar el resultado que se concentra en la opinión que sostiene que el flujo de turistas no tiene que ser limitado. La mayoría (92\%) de las personas que laboran en turismo señalaron que no debería limitarse, pero además, la opinión de personas colocadas en los otros segmentos laborales de la tipología, suministraron resultados superiores al 70\%, en cuanto a que no están de acuerdo en que se limite el número de turistas.

A partir de este rubro respecto a limitar el número de turistas, las personas censadas señalan que están en contra, además también manifiestan que la calidad de vida está asociada a la existencia de la dinámica del turismo, esto lo opinan de manera generalizada en los diferentes sectores de la tipología. Sin embargo, aparece un contraste relevante cuando repasamos las opiniones sobre la afectación al ambiente. En este punto, todos sectores de la tipología, excepto el turismo, presentan una mayoría de entrevistados que aceptan los efectos negativos de la actividad turística sobre la calidad beneficiosa del ambiente natural. Y además, en el caso del sector turismo, si bien es cierto, la mayoría dice que no afecta o maltrata la naturaleza, el porcentaje solo es mayor en un 3\%, ya que el 37\% dicen estar de acuerdo o más con esta afectación. Es decir, pese a reconocer que se dan efectos negativos en la naturaleza, también se acepta o se señala que el beneficio obtenido de la actividad, ha sido por lo general, un factor positivo.

Esta comparación nos permite conjeturar que la valoración fundamental es el peso económico que la actividad turística tiene en los hogares e individuos. Se podría señalar que el costo natural y ambiental es tolerable, siempre que el conato económico-monetario exista y tenga un peso significativo en la dinámica financiera familiar. En otras palabras, el criterio socio-económico es determinante y el tema ambiental, así como otras temáticas, podrían ser contingentes en la valoración de las decisiones respecto a las dinámicas y las actitudes referentes al accionar individual y colectivo en el escenario del turismo.

Esta última consideración ronda la evidencia de que hay posiciones contrarias en términos de las valoraciones sobre el turismo, es decir, no hay una posición rotunda respecto a una ejecución turística adaptable, por el contrario, se demuestra más bien, una forzada adaptación que no redunda, necesariamente, en beneficios sostenibles, y que tampoco garantiza el ingreso de dinero ni presenta con claridad los beneficios colectivos, más allá de ingresos específicos y utilizables. 


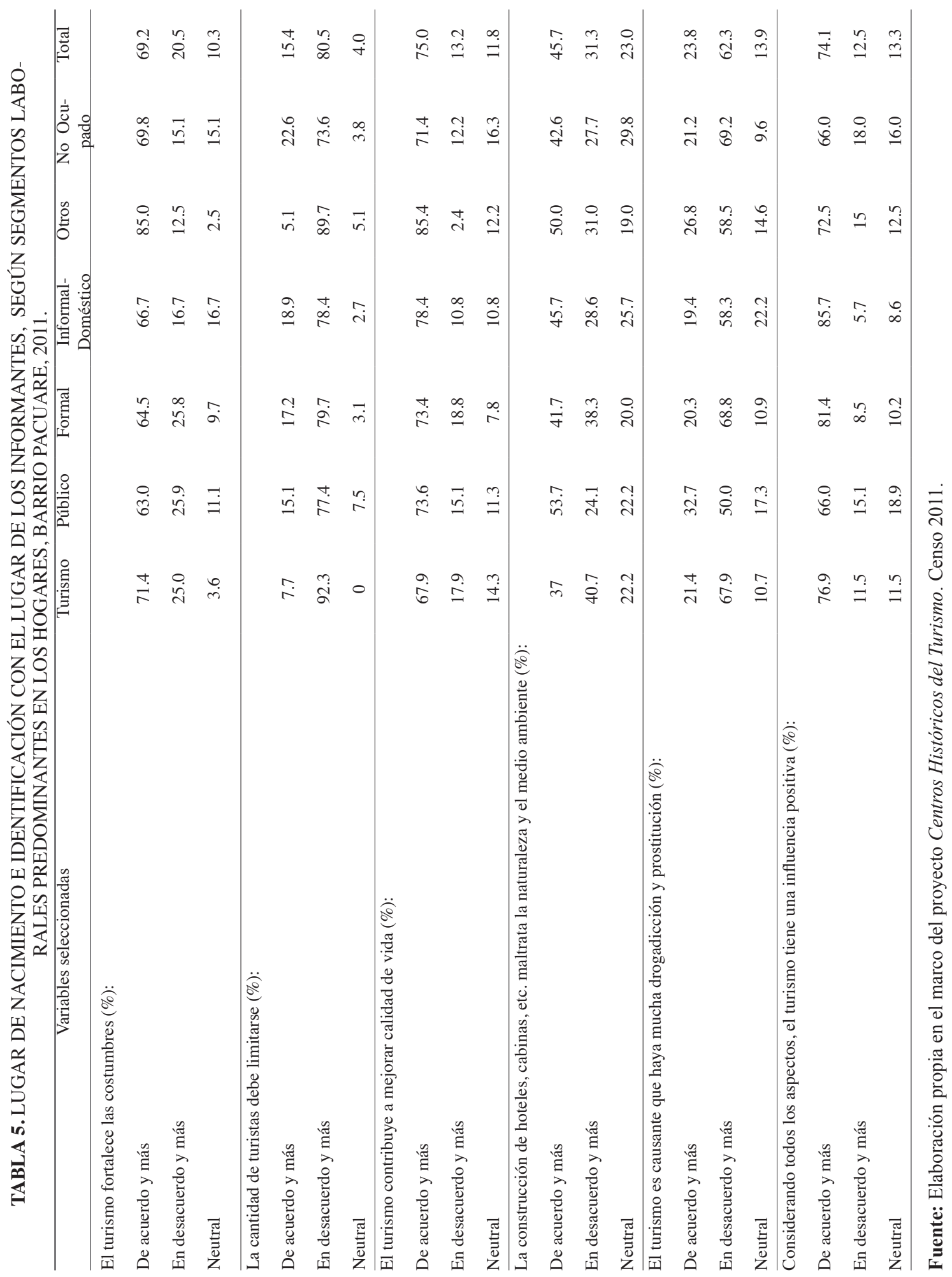




\section{CONCLUSIONES PRELIMINARES SOBRE EL TURISMO EN EL BARRIO PACUARE}

Dentro de las consideraciones generales está rescatar al turismo como una opción fundamental para un sector joven (altamente feminizado) que ha encontrado en este espacio una posibilidad para atender las demandas individuales y las derivadas de la condición de jefaturas de hogar.

Además, rescatar la consideración sobre la opinión extendida en los distintos segmentos estudiados que apoya y ve como positiva la incorporación, incluso sin restricciones de cantidad, de más turistas dentro de la zona socio-geográfica de Pacuare. Asimismo se aprecia una diferenciación en términos de las jefaturas de familia, en el caso del segmento Turismo y en el Informal-Doméstico (este último esperable por la condición de madres solteras, de muchas empleadas de este sector), en los que las mujeres son las que primordialmente llevan las jefaturas en los hogares, aspecto que difiere del resto de los segmentos laborales.

De forma muy sucinta e inicialmente, podemos observar una percepción (sin entrar en una discusión teórica sobre este concepto) positiva y de apoyo al turismo, sin que se pueda establecer si las personas que manifiestan esta opinión están del todo al tanto de las situaciones conflictivas y las consecuencias de éstas. Además, es pertinente valorar las posibles contradicciones entre el pensar, la necesidad y el hacer, que pueden estar afrontando los habitantes de la zona, ya que pese a poder detectar contradicciones entre lo que hacen y el medio ambiente y cultural (con niveles negativos para esos rubros), se ven en la necesidad de continuar con dicha lógica productiva. Por otra parte, destaca que en la opinión expresada en el censo y las manifestaciones públicas se declara el apoyo a la actividad turística. Dado lo anterior, es importante mantener un matiz de prudencia crítica respecto a los datos y lo que sugieren, así como lo que potencian en el análisis respecto a las subjetividades.

\section{CITAS Y NOTAS}

1. Proyecto adscrito a la Vicerrectoría de Investigación de la Universidad de Costa Rica. Número de inscripción: 211-B0-246.

2. Otros: esta categoría involucra trabajos o actividades que no se pudieron incluir o como nueva categoría o dentro de las cuatro categorías básicas antes señaladas. En el caso de Puntarenas se agrega una categoría más a las básicas, la Pesca. Sin embargo en el caso de Limón esta categoría no surgió.

3. La categoría No ocupados funciona exclusivamente para la tipología en el caso de los hogares. 
4. Personas del país visitando zonas del mismo país, y creando tanto rutinas de sitios predilectos, como rotando los diferentes destinos que se fueron presentando y desarrollando como zonas turísticas. Ejemplo de esto fueron los puertos de Limón y Puntarenas, así como la provincia de Guanacaste.

5. La idea de satisfacción internacional no se discute, sino, más bien se postula como una situación crítica que a nivel crítico se exponen en otros trabajos y reflexiones.

6. Uno de los textos clásicos de F. Tönnies se titula precisamente: Comunidad y Sociedad, (en alemán: Gemeinschaft und Gesellschaft) en 1887.

7. El análisis de Tönnies, 1887 es mucho más profundo y denso, pero lo omitimos para posicionar el documento de matizada y con una exposición sintética de los datos.

8. Clarificar también, si bien algunas corrientes sociológicas unen la idea de comunidad con las corrientes organicistas, o el contractualismo. En este documento no lo llevamos por ese camino (y de hacerlo se unificaría más con una versión contractualista, pero amortiguada por ciertas aclaraciones), y dejamos el concepto de comunidad, como una forma organizativa cultural, que tiene lógicas internas, así como convivencias con el exterior, y que se orienta a la construcción de patrones de vivencia económica creativa, que no deja de lado las relaciones jerárquicas, y en conexión con su vivencia geográfica.

9. No es que no existieran fracturas antes, sino que se han llevado a cabo la generación de otra modalidad de quiebre social, o reacomodo social desde el impacto global comercial del turismo.

\section{BIBLIOGRAFÍA}

Bialakowsky,A.(2010). Comunidad y sentido en la teoría sociológica contemporánea: las propuestas de A. Giddens y J. Habermas. Papeles del CEIC. Argentina: Universidad de Buenos Aires.

Cordero, A. (2011). Los viejos y olvidados centros históricos del turismo: algunas evidencias para el caso costarricense. ¿Es posible otro turismo? Su realidad centroamericana, nueve casos de estudio. Vol. 2 (1).

García, P., \& Lepe, I. (2009). Sostenibilidad turística. Un análisis del marco jurídico turístico costarricense y del escenario turístico de la provincia de Limón, Costa Rica. Inter Sedes, X(19), 39-61. 
Pérez, J. P., Andrade, K., y Bastos, S. (2004). La estructura social ante la globalización. Procesos de reordenamiento social en Centroamérica durante la década de los 90. Costa Rica: FLACSO.

Poviñan, A. (1949). La idea sociológica de "comunidad". Actas del Primer Congreso Nacional de Filosofía. Argentina: Universidad de Buenos Aires.

Molina, P., Jiménez, L.D. y Román, L.A. (2004). Análisis y evaluación de las fuentes de financiamiento y crédito para la microempresa turística: estudio de caso de Cahuita y cantón central de Limón. Tesina para optar por el grado de bachiller en Turismo Ecológico. Costa Rica: Universidad de Costa Rica. Sede Regional Limón.

\section{ACERCA DEL AUTOR}

Pablo Carballo Chaves: Maestro en Ciencias Sociales (FLACSO). Licenciado en Sociología por la Universidad de Costa Rica. Docente e Investigador de la Escuela de Sociología de la Universidad de Costa Rica. Investigador del proyecto: "Centros Históricos del Turismo". 